\title{
Adhesive Capsulitis. Is it possible in Childhood?
}

\author{
C. Modesto ${ }^{1}$, E. Crespo ${ }^{1}$, C. Villas ${ }^{2}$, and D. Aquerreta ${ }^{3}$ \\ ${ }^{1}$ Pediatric Department, ${ }^{2}$ Orthopedic Surgery Department and ${ }^{3}$ Radiology Department, \\ Clínica Universitaria de Navarra, Spain
}

\begin{abstract}
Adhesive capsulitis affecting shoulders has been extensively studied by orthopedic surgeons, but few reports have been published on this condition when it affects other joints (hips, ankles). To our knowledge no pediatric cases have been reported. As its treatment requires prolonged physiotherapy and sometimes manipulation, a correct diagnosis is essential. We would like to emphasize the importance of having this condition in mind when one is regarding a differential diagnosis, also in children.
\end{abstract}

\section{KEY WORDS}

Hip, adhesive capsulitis, childhood

Correspondence: C. Modesto, Pediatric Department, Clínica Universitaria de Navarra, Aptdo 4209, Pamplona, SP-31080 Navarra, Spain

\section{INTRODUCTION}

Adhesive capsulitis was first described by Putnam in 1882 (1). It is characterized by limited movement of the joint and pain at the extremes pf motion. During many years the terms "frozen shoulder" and "adhesive capsulitis" were accepted as synonyms. In 1987, Neviaser (2) published a brief review of this condition where the necessity to differentiate between true adhesive capsulitis and stiff, painful shoulder was emphasized. In this article, true adhesive capsulitis was defined as an inflammatory reaction in the capsule and synovium of the shoulder that subsequently leads to the formation of adhesions and attachment of the capsule at the anatomic neck of the humerus. It follows different stages from the pre-adhesive stage (stage I), characterized by minimal limitation of motion and fibrinous synovial inflammation, to stage IV (the chronic stage), in which adhesions are fully mature. 
Few reports have been published regarding the involvement of different joints other than the shoulder $(3,4)$. Hips and ankles can be affected by this condition, generally following a repeated minor trauma. Arthrography is considered essential when adhesive capsulitis is suspected. This will show a reduced volume of synovial fluid (usually less than $8 \mathrm{ml}$ ) and an increased intra-articular pressure (4).

\section{CASE REPORT}

A 10 year old girl was referred to our hospital after being admitted into another center for two months. The reason for the referral was the presence of an almost frozen painful left hip. A trial with NSAIDs had been performed before referral without satisfactory results.

During the previous summer this patient had been training for horse-riding for two months. Symptoms began shortly after that in the form of an insidious pain in the left hip. The condition worsened in the following months, both in pain and restriction of movement. At the time of her visit to our hospital she could not walk, hip external rotation and extension were virtually non existent, and abduction and internal rotation were rather limited. Her right hip presented slight limited external rotation.

Blood test showed normal WBC, hemoglobin, ESR, liver and renal function tests, negative RF, negative ANA and normal immunoglobulins. The tuberculin test was negative. X-rays showed no abnormalities apart from a mild osteoporosis affecting her left hip. MRI was normal (Fig. 1). A Tc ${ }^{99}$ scan proved to be positive showing a "hotter" diffused area surrounding the left hip.

Finally, because the hip is only rarely affected as a single joint in JRA, and because of the presence of an almost immobile joint, the decision to perform a synovial biopsy was made. Surgical findings proved to be the clue for diagnosis. First, under general anesthesia, the left hip continued to show quite a restricted external rotation. An attempt to mobilize the joint was performed achieving a slight increased range of motion. Some "cracking" was noticed by the surgeon during the manipulation. Second, synovial fluid did not flow from the joint when the capsule was cut. The capsule was tethered to the femoral head. Synovial membrane was macroscopically normal. Pathology also showed no abnormal findings in the synovium at this point. Unfortunately, no capsular tissue was sent for microscopic analysis.

Once the diagnosis of adhesive capsulitis was suspected, intensive physiotherapy and anti-inflammatory treatment were started. We used indometacin $(2 \mathrm{mg} / \mathrm{kg} / \mathrm{day})$ in three divided doses. The patient was sent home after 5 days of hospitalization and has been closely followed in our clinic since then. After two months the range of motion improved noticeably, even though external rotation was still mildly limited. Functionally, the girl became completely autonomous, being able to fully participate in school activities again. After a year of follow-up, she presents a painless left hip with full range of motion, being off anti-inflammatory treatment for six months. 


\section{DISCUSSION}

Arthritis of the hip is one of the most challenging problems in Pediatric Rheumatology. The reason for this is the: special anatomy and vascularization of this joint. Congenital or septic processes (5) should be ruled out before believing in an inflammatory condition.

Taking into account the clinical course of the disease and the results of X-rays and MRI, the diagnosis of septic arthritis, bone tumor and congenital malformation were excluded. Because adhesive capsulitis was not suspected at first, synovial biopsy was considered essential. Surgical findings helped us to make a definitive diagnosis. Normal pathology of the synovial membrane was in agreement with the fact that we were not facing an inflammatory synovitis, such as JRA.

As mentioned above, adhesive capsulitis has been reported only occasionally outside the shoulder. There is no data about its incidence in the pediatric population. The idea we would like to emphasize is that adhesive capsulitis should be considered in the differential diagnosis of prolonged hip pain, especially if there is a history of repeated minor trauma, not only in adults but also in children. When this condition is suspected, arthrography is the most effective means of diagnosis (2).

Making a correct diagnosis does not only have an academic importance. Adhesive capsulitis is a long-term condition that may require physiotherapy for months. For this reason an individual program should be planned, ideally at home (1).

With our limited experience, we think that anti-inflammatories are helpful for the treatment. In our opinion, these drugs reduce the pain and effectively facilitate the role of physiotherapy, which is really the cornerstone of the treatment. Parents should be reassured about the self-limiting character of this condition, and its usual good response to the therapy.

In order to know the true incidence of adhesive capsulitis throughout childhood, a multicenter analysis is required. This study will help us, as well, to increase our knowledge of the nature of this condition in joints different from the shoulder.

\section{REFERENCES}

1. Thornhill ThS: Shoulder Pain. In: Kelley WN, Harris ED, Ruddy S, Sledge CB, eds. Textbook of Rheumatology. Philadelphia: WB Saunders Co. 1993: 417-40.

2. Neviaser RJ, Neviaser ThJ. The Frozen Shoulder. Diagnosis and Management. Clin Orthop 1987; 223: 59-63.

3. Lequesne M, Becker J, Bard M, Witvoet J, Postel M. Capsular Constriction of the Hip: Arthrographic and Clinical Considerations. Skeletal Radiol 1981; 6: 1-10.

4. Griffiths HJ, Utz R, Burke J, Bonfiglio Th. Adhesive Capsulitis of the Hip and Ankle. AJR Am J Roentgenol 1985; 144: 101-5.

5. Cassidy JT, Petty RE. Juvenile Rheumatoid Arthritis. In: Cassidy JT, Petty RE, eds. Textbook of Pediatric Rheumatology. Third Edition. Philadelphia; WB Saunders Co. 1995: 133-223. 


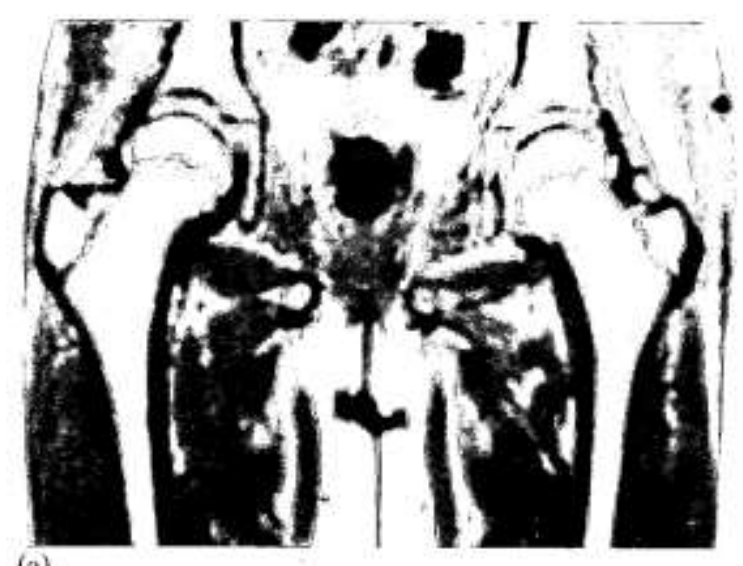

(a)

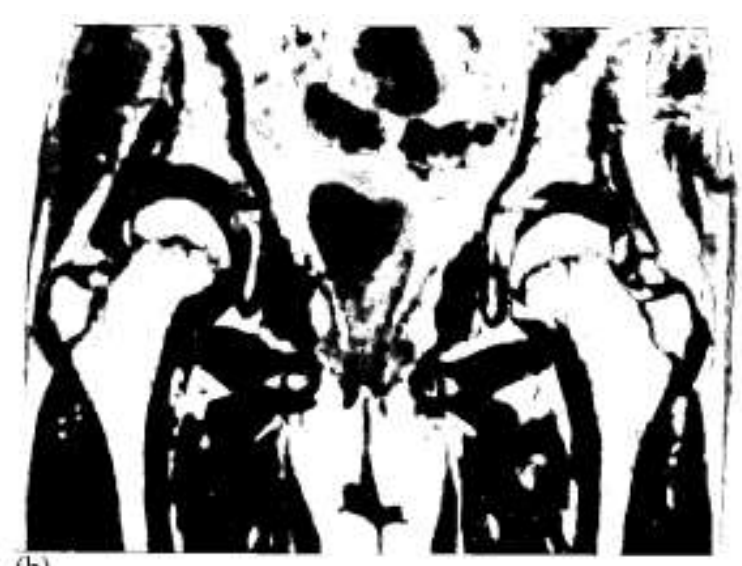

(b)

Figura 1. MRI of both hips, showing no abnormalities. (a) T1 weighted; (b) with gadolinium. 